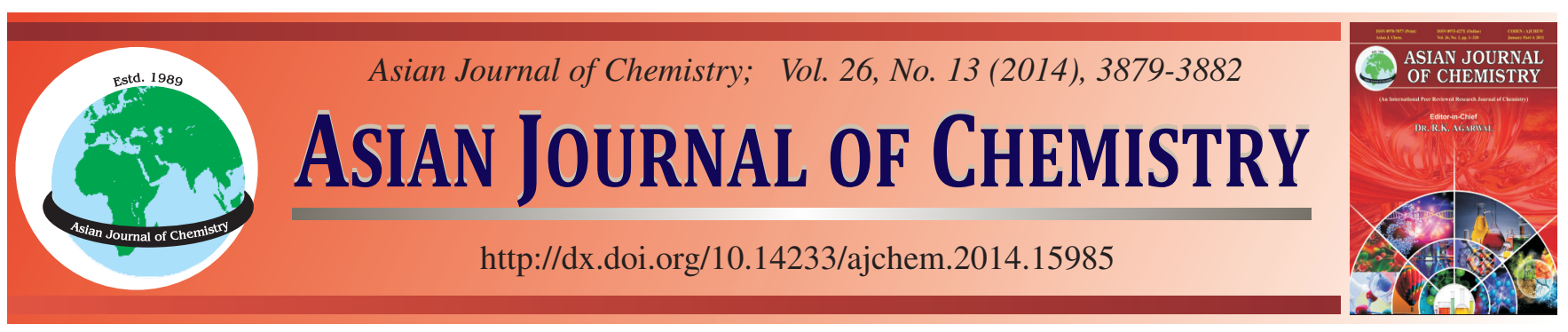

\title{
Electrochemical Reductive Dehalogenation of ortho-Halogenated Phenols on Ag Electrode by in situ FTIR
}

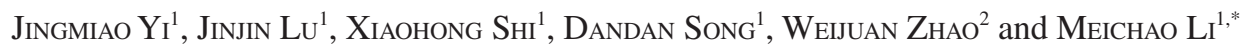

${ }^{1}$ Research Center of Analysis and Measurement, College of Chemical Engineering and Materials Science, State Key Laboratory Breeding Base of Green Chemistry-Synthesis Technology, Zhejiang University of Technology, Hangzhou 310032, P.R. China ${ }^{2}$ Zhejiang Research Institute of Chemical Industry, Ltd., Hangzhou 310023, P.R. China

*Corresponding author: E-mail: limc@zjut.edu.cn

\begin{abstract}
Electrochemical reductive dehalogenation reactions of ortho-halogenated phenols, namely, $o$-iodophenol (OIP), $o$-bromphenol (OBP) and $o$-chlorophenol (OCP) on Ag electrode in alkaline medium have been studied by in situ FTIR combined with cyclic voltammetry and computational calculations. The Ag electrode showed a high electrochemical activity for dehalogenation reactions of OBP and OIP in contrast with OCP under the similar conditions and the dehalogenation potential of OIP was more positive than OBP, reflecting more facile reduction of OIP on Ag electrode. On the basis of in situ FTIR of OCP on Ag electrode, it was not obvious and the electrochemical reduction reaction was quite weak. Therefore, the order of electrochemical reductive dehalogenation was OIP > OBP > OCP.
\end{abstract}

Keywords: $o$-Iodophenol, $o$-Bromphenol, $o$-Chlorophenol, Electrochemical reductive dehalogenation, in situ FTIR.

\section{INTRODUCTION}

With the development of chemical industry, the halogenated phenols are widely used in the synthesis of pharmaceutical and agrochemical products and have been frequently found in many surface and ground waters. It is well known that the halogenated phenols are toxic and anti-degradable, due to the aromatic ring and halogen contained in their structure $^{1,2}$. Therefore, it is highly desirable to develop new treatment technologies for the decomposition of halogenated phenols in wastewater.

Traditional treatment processes such as biological, photochemical and chemical are not quite effective for the dehalogenation of the halogenated phenols. Recently, electrochemical reductive dehalogenation has been widely studied. With low environmental contamination and high selectivity, it has successfully developed to be the new and one of potential method degradation of halogenated phenols. Halide ions could be released by electrochemical reductive dehalogenation and then phenols can be disposed completely combined with other methods.

However, electrochemical reductive dehalogenation of halogenated phenols is affected by many factors, such as the electrolyte, electrode material, etc $^{3-5}$. According to the literatures, Ag electrode is proved to be one of the perfect electrode materials and shows good performance on electroreductive dehalogenation ${ }^{6,7}$. In addition, the halogen substituents and different subpositions have effect on the electrochemical dehalogenation reaction. In this paper, $o$-iodophenol(OIP), $o$ bromphenol (OBP) and $o$-chlorophenol (OCP) were chosen as the model molecules of the ortho-halogenated phenols and the Ag electrode was chosen as the working electrode. The experiments were performed in aqueous solution in order to search for a better water treatment technology for a polluted water body. The aims of our work were to: (i) investigate the electrocatalytic activity of Ag electrode for electrochemical reductive dehalogenation of the ortho-halogenated phenols, (ii) examine the mechanism of electrochemical reductive dehalogenation of the ortho-halogenated phenols on Ag electrode using in situ FTIR technique, (iii) examine the influence of the halogen substituents on the ortho-halogenated phenols using in situ FTIR technique combined with cyclic voltammetry and computational calculations.

\section{EXPERIMENTAL}

The concentration of $o$-iodophenol(OIP), $o$-bromphenol (OBP) and $o$-chlorophenol (OCP) was $0.05 \mathrm{~mol} \mathrm{~L}^{-1}$. $\mathrm{NaOH}$ $\left(0.6 \mathrm{~mol} \mathrm{~L}^{-1}\right)$ was used as the supporting electrolyte. All reagents were of analytical reagent grade and used as received. All solutions were prepared using ultra-pure water with a resistivity of 18.2 MW obtained from Millipore-Q system.

Cyclic voltammetry: Cyclic voltammetric measurements were performed using EG\&G potentiost/galvanostat Model 
263 at room temperature. The electrochemical reductive reactions of OIP, OBP and OCP were carried out in $\mathrm{NaOH}$ solution by cyclic voltammetry between $0 \mathrm{~V}$ and $-1.8 \mathrm{~V}$ at a scan rate of $50 \mathrm{mV} \mathrm{s}^{-1}$. Ag electrode with the geometric area of $0.2 \times 0.5$ $\mathrm{cm}^{2}$ was used as the working electrode, a platinum sheet $(1.5 \times$ $\left.2.0 \mathrm{~cm}^{2}\right)$ as the counter electrode and a saturated calomel electrode (SCE) as the reference electrode.

in situ FTIR experiments: in situ FTIR spectroscopic experiments were carried out on Nicolet 670 FTIR spectrometer equipped with a MCT-A detector cooled with liquid nitrogen. The spectroelectrochemical cell was provided with a $\mathrm{CaF}_{2}$ disk window (diameter $32 \mathrm{~mm}$ and thickness $2 \mathrm{~mm}$ ). The working electrode was Ag electrode with the surface pressed against the $\mathrm{CaF}_{2}$ disk window to form a thin layer for IR measurements. Silver disk electrode (diameter $6 \mathrm{~mm}$ ) was used as the working electrode, which was polished by the metallographic sand papers. A platinum sheet $\left(1.5 \times 2.0 \mathrm{~cm}^{2}\right)$ was used as the counter electrode and the SCE was used as the reference electrode. To acquire in situ FTIR data, 200 interferograms were collected at each potential at a resolution $8 \mathrm{~cm}^{-1}$.

Computational calculations: All calculations in this work were carried out using GAUSSIAN03W program within the framework of density functional theory. The B3LYP methodBecke's three parameter hybrid functional using the LYP correlation functional was employed for geometry optimization and electronic property calculations. In all cases, the 6-31G(d) basis set was used ${ }^{8,9}$.

\section{RESULTS AND DISCUSSION}

Cyclic voltammetry studies: Cyclic voltammograms of OIP, OBP and OCP in $\mathrm{NaOH}$ solution on $\mathrm{Ag}$ electrode respectively between $0 \mathrm{~V}$ and $-1.8 \mathrm{~V}$ at a scan rate of $50 \mathrm{mV} \mathrm{s}^{-1}$ were shown in Fig. 1. The voltammogram of the Ag electrode in $0.6 \mathrm{M} \mathrm{NaOH}$ supporting electrolyte alone was given in Fig. 1 . During the cathodic potential scanning in Fig. 1 (a), the reduction of OIP began at approximately $-900 \mathrm{mV}$. With the potential shifting to more negative, the reduction current increased gradually and a characteristic irreversible peak at about -1.15 $\mathrm{V}$ with the current peak about $1.73 \mathrm{~mA}$ was observed. It indicated that $\mathrm{Ag}$ electrode showed a good electrocatalytic activity for OIP and the reaction was irreversible. Fig. 1 (b) showed the electrochemical reduction reaction of OBP could happen on the Ag electrode surface too. But the reduction peak current was lower than OIP under the similar conditions. Voltammogram of OCP on Ag electrode was shown in Fig. 1 (c), except for the peak of hydrogen evolution, almost no obvious peaks assigned to electrochemical reduction of OCP on Ag electrode could be observed. Therefore, electrochemical reduction of OCP on Ag electrode was quite weak.

in situ FTIR spectroscopic studies: in situ FTIR is one of the important techniques to analyze the electrochemical reaction. The resulting spectrum of in situ FTIR recorded was defined as the potential-difference spectra ${ }^{10}$ and was calculated by the following formula:

$$
\frac{\Delta R}{R}=\frac{R\left(E_{s}\right)-R\left(E_{R}\right)}{R\left(E_{R}\right)}
$$
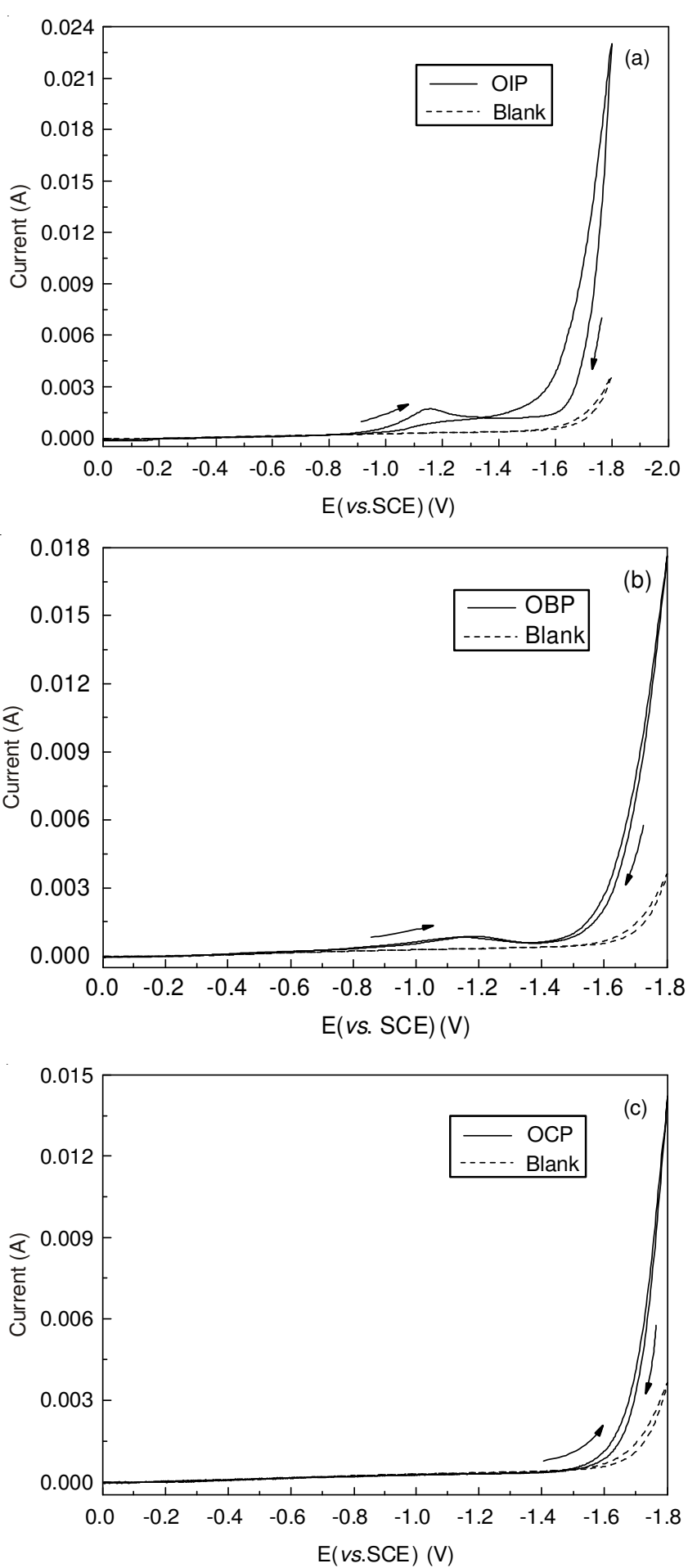

Fig. 1. Cyclic voltammograms for the electrochemical reduction of (a) OIP, (b) OBP and (c) OCP on Ag electrode at $50 \mathrm{mV} \mathrm{s}^{-1}$. (---) $\mathrm{Ag}$ in the supporting electrolyte

where $R\left(E_{s}\right)$ and $R\left(E_{R}\right)$ are single-beam spectra collected respectively at the sample potential $\left(\mathrm{E}_{\mathrm{s}}\right)$ and the reference potential $\left(E_{R}\right)$. Negative-going bands in the resulting spectra indicate the formation of intermediates or products, while positivegoing bands denote the consumption of reactants.

in situ FTIR spectra of OIP: Fig. 2 showed in situ FTIR spectra obtained during electrochemical reductive reaction of OIP on Ag electrode. The reference spectrum was acquired at $0 \mathrm{mV}$, then the $\mathrm{Ag}$ cathode was polarized from -200 to -1400 
$\mathrm{mV}$ at a constant modulation potential $(\Delta \mathrm{E}=-200 \mathrm{mV})$ and the sample spectra were collected. The polarization time at each potential was about $90 \mathrm{~s}$.

When the sample potentials were more positive than $-800 \mathrm{mV}$, no obvious changes could be observed on the FTIR spectra in Fig. 2 and the electrochemical reaction was very weak on the electrode surface. At approximately $-1000 \mathrm{mV}$, the spectra were dominated by three characteristic negativegoing bands at 1456, 1431 and $1303 \mathrm{~cm}^{-1}$, which were attributed to benzene framework vibration and $\mathrm{C}-\mathrm{O}$ stretching vibration of $\mathrm{OIP}^{11,12}$. It indicated that electrochemical reaction of OIP on Ag cathode occurred with a slow velocity at approximately $-1000 \mathrm{mV}$. When the potential stepped to more negative, new bands were originated in the thin layer at approximately $-1200 \mathrm{mV}$. The positive-going bands at $1575,1114 \mathrm{~cm}^{-1}$ were ascribed, respectively to $\mathrm{C}=\mathrm{C}$ stretching of aromatic ring ${ }^{11,13}$, $=\mathrm{C}-\mathrm{H}$ in-plane bending ${ }^{11,12}$. In addition, an important positivegoing band at $1006 \mathrm{~cm}^{-1}$ assigned to C-I stretching of OIP was generated from the $\mathrm{C}$-I bond breakage, indicating that deiodination reaction of OIP occurred ${ }^{11,13}$.

Meanwhile, four negative-going bands located at 1588, 1482, 1271 and $1168 \mathrm{~cm}^{-1}$ can be observed clearly in Fig. 2, which were related to ring vibration of phenol anion, benzene framework vibration ${ }^{11-13,15}$, C-O stretching vibration in aromatic ring ${ }^{11,12,16}$ and $\mathrm{C}-\mathrm{H}$ in plane bending vibration of phenol anion in aromatic ring ${ }^{11,12,15}$, respectively. But the reaction was weak and the negative-going peaks were not quite obvious. When the potential stepped to more negative, the rate of deiodination reaction increased and the amount of monosubstituted phenol anion increased, while the intensities of 1588 and $1168 \mathrm{~cm}^{-1}$ become stronger and stronger.

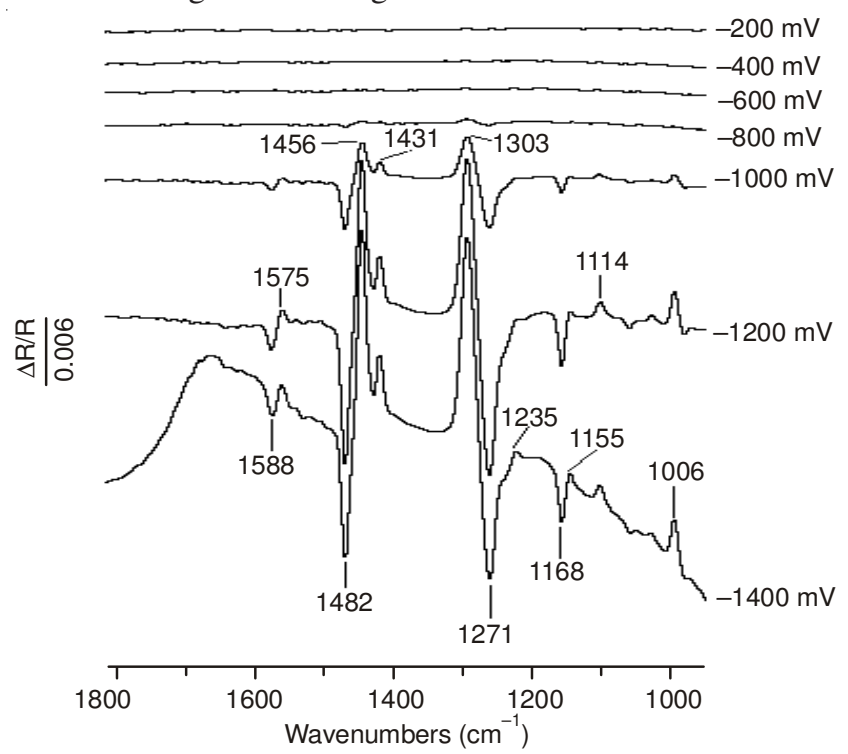

Fig. 2. in situ FTIR spectra collected during electrochemical reduction of OIP on $\mathrm{Ag}$ electrode in $\mathrm{NaOH}$ solution

On the basis of related literatures and experimental results $^{15-18}$, the mechanism of electrochemical reduction of OIP on $\mathrm{Ag}$ electrode can be represented as a sequence of electron additions and halogen expulsions (Scheme-I). $o$-Iodophenol was electroreduced to its free radical ion after receiving an electron. With release of iodine ion from OIP free radical at the negative potentials, the phenolate anion was obtained finally.

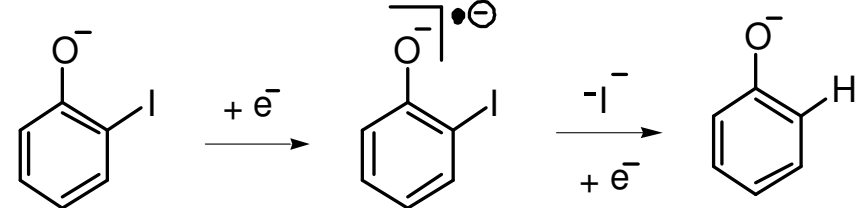

Scheme-I: Mechanism of electrochemical reduction of OIP on Ag electrode

in situ FTIR spectra of $o$-bromophenol on Ag electrode: Fig. 3 showed in situ FTIR spectra obtained during electrochemical reduction of OBP on Ag electrode. When the sample potentials were more positive than $-800 \mathrm{mV}$, no obvious changes could be observed on the FTIR spectra in Fig. 3 and electrochemical reaction of OBP was very weak on the surface of electrode. When the potentials stepped to more negative, especially at $-1400 \mathrm{mV}$, the corresponding infrared signals of positive-going and negative-going bands changed significantly. It indicated that the intensities of electrochemical reductive reaction were increasing on the surface of $\mathrm{Ag}$ electrode. The important positive-going band at $1021 \mathrm{~cm}^{-1}$ assigned to $\mathrm{C}-\mathrm{Br}$ stretching vibration was generated from the $\mathrm{C}-\mathrm{Br}$ band breakage, indicating that debromination reaction of OBP occurred on the Ag electrode. Positive-going bands at 1575, 1463, 1437 $\mathrm{cm}^{-1}$ were related to $\mathrm{C}=\mathrm{C}$ framework vibration of aromatic ring in OBP. And positive-going bands at 1306, $1232 \mathrm{~cm}^{-1}$ were assigned to $\mathrm{C}-\mathrm{O}$ stretching vibration of OBP and 1155 , $1114 \mathrm{~cm}^{-1}$ assigned to $=\mathrm{C}-\mathrm{H}$ in-plane bending of aromatic ring. These positive-going bands indicated that OBP was reduced with the electrochemical reduction. Meanwhile, there were four negative-going bands at 1588, 1482, 1274 and $1168 \mathrm{~cm}^{-1}$ in Fig. 3, which were related to ring vibration of phenol anion ${ }^{12}$, benzene framework vibration, $\mathrm{C}-\mathrm{O}$ stretching vibration in aromatic ring ${ }^{11,12,16}$ and $\mathrm{C}-\mathrm{H}$ in plane bending vibration of phenol anion in aromatic ring ${ }^{11,12,15}$, respectively.

So the mechanism of electrochemical reduction of OBP on $\mathrm{Ag}$ electrode could also be represented as a sequence of electron additions and halogen expulsions, which is similar with OIP. Comparative analysis of Figs. 2 and 3 showed that the electrochemical reductive dehalogenation reaction of OIP and OBP could occur on Ag electrode. But the potential for deiodination reaction of OIP was more positive than debromination reaction of OBP. It indicated that deiodination reaction of OIP is easier than debromination reaction of OBP. In addition, negative-going bands in Fig. 2 were stronger than those in Fig. 3, so the deiodination reaction of OIP is more active.

in situ FTIR spectra of $o$-chlorophenol on Ag electrode: Fig. 4 showed in situ FTIR spectra obtained during electrochemical reduction of OBP on Ag electrode. When potential at $-1000 \mathrm{mV}$, no obvious changes could be observed on the FTIR spectra in Fig. 4. When potential at $-1400 \mathrm{mV}$, the positivegoing bands at 1575, 1469, 1437, 1303, 1237, 1156, 1122 and $1030 \mathrm{~cm}^{-1}$ which were related respectively to aromatic ring framework vibration of OCP, C-O stretching vibration on aromatic ring, $\mathrm{C}-\mathrm{H}$ in-plane bending on aromatic ring and $\mathrm{C}-\mathrm{Cl}$ stretching vibration were observed. However, the negative-going bands were very weak and could hardly be observed. It could be inferred that Ag exhibited a weak electrochemical reduction activity of $\mathrm{OCP}$ in $\mathrm{NaOH}$ solution. 


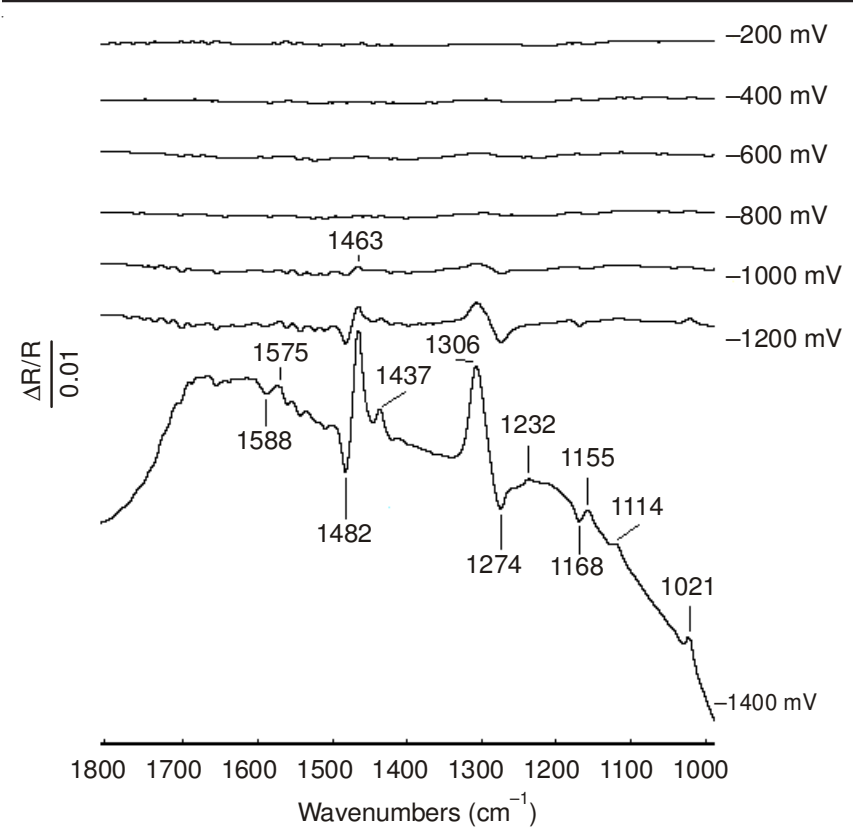

Fig. 3. in situ FTIR spectra collected during electrochemical reduction of OBP on $\mathrm{Ag}$ electrode in $\mathrm{NaOH}$ solution
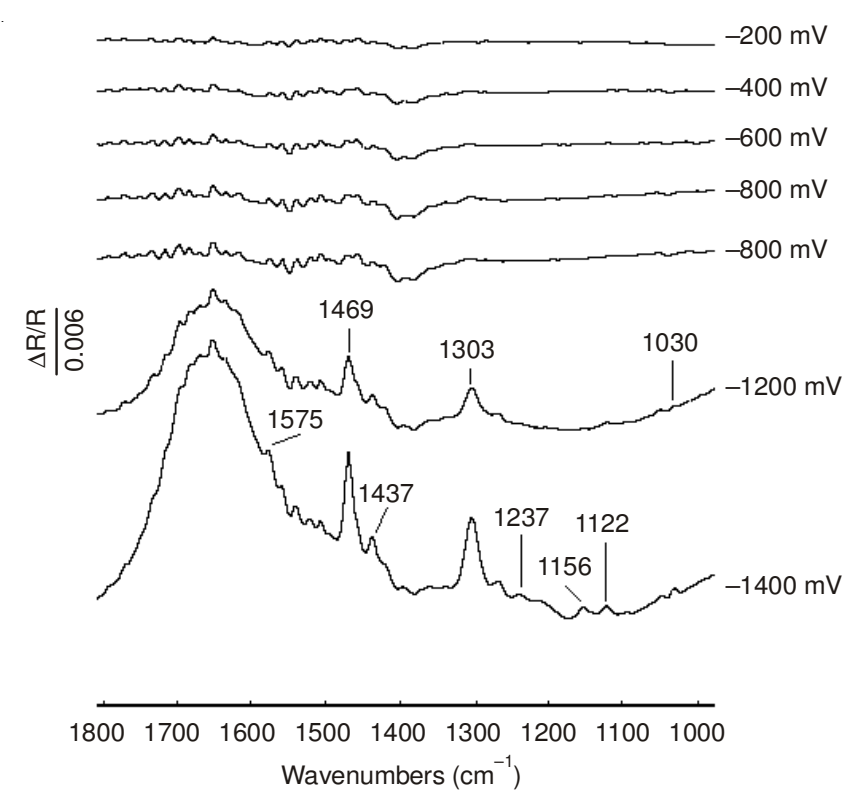

Fig. 4. in situ FTIR spectra collected during electro-reduction of OCP on Ag electrode in $\mathrm{NaOH}$ solution

The order of halogen ions to take off from halogenated phenols might be related to the electronegativity and ionic radius of the halogens. The electronegativity of $\mathrm{I}, \mathrm{Br}$ and $\mathrm{Cl}$ increased in turn and their ionic radius decreased. So the bond energy of C-X increased from OCP to OIP. Meanwhile, the bond dissociation energies of $\mathrm{C}-\mathrm{X}$ bonds in ortho-halogen phenols were calculated ${ }^{19}$. Among the three bands, the bond dissociation energy of $\mathrm{C}-\mathrm{Cl}$ was $0.1572 \mathrm{au}$, which is the highest. The energy of C-Br was 0.1472 au and C-I was 0.0151 au. It indicated that iodide ion was the easiest to take off from the aromatic ring, while the chlorine ion was the most difficult.

\section{Conclusion}

Electrochemical reductive dehalogenation reactions of OIP, OBP and OCP on Ag electrode have been studied in alkaline medium by the use of in situ FTIR. The results showed that deiodination reaction of OIP was easy to take place on $\mathrm{Ag}$ electrode. But the reaction current of OBP is lower than OIP while the dehalogenation potential was more negative than OIP under the same conditions. While the electrochemical reductive reaction of OCP was quite weak on Ag electrode. Therefore, the order of electrochemical reductive dehalogenation was OIP > OBP > OCP. The results of in situ FTIR were in good agreement with cyclic voltammetry and computational calculations.

\section{ACKNOWLEDGEMENTS}

This work was financially supported by the State Key Development Program for Basic Research of China (973) (Grant No. 2012CB722604) by the National Natural Science Foundation of China (Grant No. 21206147) and the Key Innovation Team Foundation of Zhejiang Province (2009R5000221). The financial support is gratefully acknowledged.

\section{REFERENCES}

1. R.T. Benedict, H.M. Stapleton, R.J. Letcher and C.L. Mitchelmore, Chemosphere, 69, 987 (2007).

2. H. Cheng, K. Scott and P.A. Christensen, Electrochim. Acta, 49, 729 (2004).

3. R.D. Coteiro and A.R.D. Andrade, J. Appl. Electrochem., 37, 691 (2007).

4. H. Cheng, K. Scott and P.A. Christensen, J. Electrochem. Soc., 150, D25 (2003).

5. C. Schuth and M. Reinhard, Appl. Catal. B, 18, 215 (1998).

6. C. Bellomunno, D. Bonanomi, L. Falciola, M. Longhi, P.R. Mussini, L.M. Doubova and G. Di Silvestro, Electrochim. Acta, 50, 2331 (2005).

7. M. Altarawneh, B.Z. Dlugogorski, E.M. Kennedy and J.C. Mackie, J. Phys. Chem. A, 112, 3680 (2008).

8. M. Altarawneh, M.W. Radny, P.V. Smith, J.C. Mackie, E.M. Kennedy and B.Z. Dlugogorski, Appl. Surf. Sci., 254, 4218 (2008).

9. S. Rondinini, P.R. Mussini, M. Specchia and A. Vertova, J. Electrochem. Soc., 148, D102 (2001).

10. L. Tian, J.T. Li, J.Y. Ye, C.H. Zhen and S.G. Sun, J. Electroanal. Chem., 662, 137 (2011).

11. X.Y. Jing, Guidance on the Application of Infrared Spectroscopy, Tianjin Science and Technology Press: Tianjin, pp 181-185, 200-202 (1992).

12. J.X. Xie, J.B. Chang and X.M. Wang, The Application of Infrared Spectroscopy in Organic Chemistry and Medicinal Chemistry; Science Press: Beijing, pp 161-163 (2002).

13. G.Y. Popova, Y.A. Chesalov and T.V. Andrushkevich, React. Kinet. Catal. Lett., 83, 353 (2004).

14. K.M. Su, T.Y. Pan and Y.L. Zhang, Spectrum Analysis Method; East China University of Science and Technology Press: Shanghai, pp 106-107 (2002).

15. R. Chetty, P.A. Christensen, B.T. Golding and K. Scott, Appl. Catal. A, 271, 185 (2004).

16. S. Scirè, C. Crisafulli, R. Maggiore, S. Minicò and S. Galvagno, Appl. Surf. Sci., 93, 309 (1996).

17. W.C. Wu, L.F. Liao, C.F. Lien and J.L. Lin, Phys. Chem. Chem. Phys., 3, 4456 (2001).

18. A.A. Isse, L. Falciola, P.R. Mussini and A. Gennaro, Chem. Commum. 344 (2006).

19. Y.R. Luo, Comprehensive Handbook of Chemical Bond Energies, CRC Press, Boca Raton, FL (2010). 